SAND94-1235

Distribution

Unlimitied Distribution

Category UC-510

Printed September, 1994

\title{
A Review of Technology for Verification of Waste Removal from Hanford Underground Storage Tanks (WHC Issue 30)
}

\author{
Siegfried Thunborg \\ Intelligent Systems Department I \\ Sandia National Laboratories \\ Albuquerque, NM 87185-5800
}

\begin{abstract}
Remediation of waste from Underground Storage Tanks (UST) at the Hanford Waste storage sites will require removal of all waste to a nearly clean condition. Current requirements are $99 \%$ clean. In order to meet remediation legal requirements, a means to remotely verify that the waste has been removed to sufficient level is needed. This report discusses the requirements for verification and reviews major technologies available for inclusion in a verification system. The report presents two operational scenarios for verification of residual waste volume. Thickness verification technologies reviewed are Ultrasonic Sensors, Capacitance Type Sensors, Inductive Sensors, Ground Penetrating Radar, and Magnetometers. Of these technologies Inductive (Metal Detectors) and Ground Penetrating Radar appear to be the most suitable for use as waste thickness sensors.
\end{abstract}

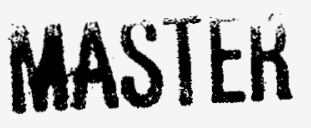




\section{Table Of Contents}

Introduction $\quad 1$

System Requirements 1

Problem Areas $\quad 1$

Positional Accuracy of Arm 2

Tool Forces $\quad 3$

Depth of Cut 3

Tank Environment 3

Tank Condition $\quad 3$

$\begin{array}{ll}\text { Tolerances } & 4\end{array}$

Operational Scenarios $\quad 4$

Sensor Control 4

PreSurvey 4

Technology $\quad 5$

Ultrasonics $\quad 5$

6

Capacitance Type Sensors

Inductive Sensors $\quad 8$

Ground Penetrating Radar 10

$\begin{array}{ll}\text { Magnetometers } & 10\end{array}$

$\begin{array}{ll}\text { Summary } & 11\end{array}$

$\begin{array}{ll}\text { Appendix A } & 12\end{array}$ 


\section{DISCLAIMER}

This report was prepared as an account of work sponsored by an agency of the United States Government. Neither the United States Government nor any agency thereof, nor any of their employees, make any warranty, express or implied, or assumes any legal liability or responsibility for the accuracy, completeness, or usefulness of any information, apparatus, product, or process disclosed, or represents that its use would not infringe privately owned rights. Reference herein to any specific commercial product, process, or service by trade name, trademark, manufacturer, or otherwise does not necessarily constitute or imply its endorsement, recommendation, or favoring by the United States Government or any agency thereof. The views and opinions of authors expressed herein do not necessarily state or reflect those of the United States Government or any agency thereof. 


\section{DISCLAIMER}

Portions of this document may be illegible in electronic image products. Images are produced from the best available original document. 


\section{A Review of Technology for Verification of \\ Waste Removal from Hanford \\ Underground Storage Tanks \\ (WHC Issue 30)}

\section{Introduction}

Remediation of waste from Underground Storage Tanks (UST) at the Hanford site will require removal of all waste to a nearly-clean condition: on the order of $99 \%$ clean. The tank initially scheduled for clean out is tank C106. It is believed this tank has up to 8 feet of solid or semisolid waste in the bottom of the tank of which the bottom 2 feet is hard waste. Removal of this waste will require the use of Waste Dislodging Tools on the end of a large remotely-operated robotic arm. In order to meet remediation legal requirements, a means to verify that the waste has been removed to sufficient levels is needed. The planned approach to this need is to confirm at the time of waste removal that the thickness of the waste remaining is within acceptable limits through the use of appropriate sensors.

This report will discuss the requirements for verification and review major technologies available for inclusion in a verification system.

\section{System Requirements}

Tri-party agreements specify that there shall be no more than 360 cubic feet of waste remaining in tank C-106 after clean out. It is believed the soft/hard waste is $8 \mathrm{ft}$. high in the bottom of tank C-106. Assuming the waste remaining after cleanout is evenly distributed in the bottom of the tank and along $8 \mathrm{ft}$. of the wall, the 360 cubic feet requirement translates to be a thickness of no more than 0.68 inches around the tank perimeter. However, it may be that the initial sluicing process conducted in the tank will adequately remove all the soft waste. In this case the 360 cubic feet requirement translates to be 0.88 inches in the bottom of the tank and along 2 feet of the walls.

For the purpose of this report, the maximum allowable residual waste thickness will be the minimum of the above; i.e., 0.68 inches.

\section{Problem Areas}

Tank cleanout to the required level using large, flexible, remotelyoperated equipment is a difficult problem. The condition of the tank's metal liner is unknown and any contact with it could cause serious problems. Any damage which occurs to the tank during remediation operation has the potential to be very costly, both in terms of repairing the damage and in terms of the down time realized during repairs and during incident investigation and resolution. 
Some of the problem areas which should be considered during development of a verification system are discussed below:

\section{Positional Accuracy of Arm}

A retrieval arm for use in tank C-106 has not yet been specified. However, procurement is currently underway for a testbed arm sized for use in UST's. The concept for this arm is a medium-sized dexterous arm on a larger gross positioner arm. Positional and control requirements for the testbed arm are as follows:

Gross Positioner

- Minimum incremented motion, 0.4 inches

- Positional accuracy, 6.0 inches

Dexterous Arm

- Repeatability, 0.1 inch

- Incremented motion, 0.1 inch

- Positioning accuracy, 0.5 inches

- Straight line motion within 0.2 inches

Overall System

- Repeatability, 0.5 inches

- Real-time tracking accuracy, 0.5 inches at constant velocity

If the arm for tank C-106 is procured to similar positional requirements, there will be several potential problem areas.

- The positional accuracy and minimum increment motion of the gross positioner would mean it would be impractical to use the gross positioner arm when trying to remove waste to less than 0.68 inches. In all likelihood, the gross positioner would be locked in place while removal was accomplished by a dexterous arm.

- A positioning accuracy of 0.5 inches for the dexterous arm insinuates the arm would require a sensor to find an actual waste surface when operating near a tank surface. Sending the arm to a surface defined in a world model would be impracticable since, for example, the tank would be assumed to be a perfect cylinder in the robot's world model, while the actual geometry can vary much more than $1 / 2$ inch due to construction tolerances. 


\section{Tool Forces}

Waste Dislodging Tools proposed for use with the retrieval arm can have reaction forces which can produce vibration and/or deflections of the arm. This displacement needs to be considered during final operations to remove the waste hardpan.

\section{Depth of Cut}

Waste Dislodging Tools (WDT) based on water jet technology are currently under development for use in retrieval operations. The depth of cut of a water jet will depend on waste characteristics. Since the waste characteristics can vary throughout the tank, the depth of cut may be hard to predict especially with cut overlap and possible WDT transverse speed variations. If it can be proven that the jet will not cut metal, depth of cut may not be a problem. However, if the jet will cut metal a sensor to detect cut depth may be required.

Pacific Northwest Laboratories has testing scheduled to determine if a high-pressure water jet will damage tank metal. The anticipated outcome of this testing is that it will not if the thin metal liner is not excessively deteriorated.

\section{Tank Environment}

Water Jet Cutters can throw out particles and a mist that can stick to a surface, an extremely tough environment for a sensor. Using a sensor for real time arm control operations will require adequate protection of the sensor to insure reliable operation. Splatter becomes less of a problem if the sensor is used to determine cut progress after or between actual cutting operations.

\section{Tank Condition}

Some tanks are known to have corroded liners and are leaking. Whether the corrosion is bad enough that the tank can be affected by the cutting action of Waste Dislodging Tools is currently unknown.

Cleanup of vertical tank walls will be complicated by the fact that the walls can have $3 \times 3$ inch angle hoop reinforcement welded to the tank wall every three feet of vertical height.

In some tanks the tank bottom is known to not be in the as-built condition. Bulges in the metal liner are known to have occurred. 


\section{Tolerances}

Cleaning of the waste to a thickness less than 0.68 inches requires consideration of all the tolerances affecting cutting operations. A dexterous arm with a straight line motion capability of 0.2 inches would require starting with a nominal goal of 0.48 inches for final waste thickness. Water Jet Cutters can cut a ragged surface. Surface detection sensors would need to average several readings to get an average waste thickness. A positioning tolerance of 0.5 inches pose problems if it is required to send the arm to a position. Position may need to be confirmed with surface contact monitoring (Touchoff).

Sensor inaccuracies are likely to be small enough to neglect.

\section{Operational Scenarios}

Verification operations can be real time operations where thickness of the residual waste is determined as cutting operations progress or verification can be an operation independent of cutting operations. Scenarios for both these methods are discussed below.

\section{Sensor Control}

In this scenario sensors on board the Waste Dislodging Tool (WDT) would detect the metal liner and through real-time control techniques, control the arm and WDT in a path to maintain the tool a set position above the tank surface. For Waste Dislodging Tools with a set dig depth this technique would insure a set residual thickness. For Waste Dislodging Tools without a set dig depth, the residual thickness would be less controllable.

The real time control technique would have the advantage of allowing the WDT to follow the tank surface even if it was bulged. The disadvantage of this technique is the necessity to insure reliable operation of the sensor under the environmental conditions in a tank during waste removal operations.

Assuming the robot arm controller would have a real-time sensor control capability, the cost of a system for this scenario will be minor compared to overall system cost: being mainly the cost and installations of the sensor system.

\section{PreSurvey}

In this scenario the operator would determine from robot arm position data that the robot arm was nearing tank metal; for discussion purposes assume within 4 inches. Removal operations with the Waste Dislodging Tool would be suspended and a survey using an appropriate metal sensor would be conducted to accurately determine the actual distance to the metal tank surface. A "touch off" would be performed to insure correct 
calibration of the arm to the waste surface. Retrieval operations with the Waste Dislodging Tool would be resumed to remove a layer of waste (assume 2 inches). The surface of the waste would be resurveyed and another layer of waste removed until the residual waste was within the allowable limit of 0.68 inches.

The primary advantage of interrupting retrieval operations to perform a survey is the metal detection sensor is not required to be operational in the environment present during retrieval operations with the Waste Dislodging Tool.

The disadvantage of this operational concept is that it increases the operation time in a tank. Assume a Waste Dislodging Tool removed 2 inches per pass. Then, for a 2 foot waste depth the tool would require 12 passes. If over the last 4 inches an additional 2 survey passes were required, the cost increase would be $2 / 12$, or $17 \%$, of the cost of retrieval operations where retrieval operation cost does not include set up, tear down, maintenance, etc., costs. For tanks containing more than 2 feet of hard waste the incremental costs become less, approaching $1 \%$ for tanks full of hard waste.

\section{Technology}

A survey of technologies that may be applicable to verifying residual hardpan waste thickness has been conducted and described in the following sections. The technologies presented are: Ultrasonics, Capacitance Type Sensors, Inductive Sensors, Ground Penetrating Radar, and Magnetometers.

\section{Ultrasonics 1}

Ultrasonics is a viable solution for non-contact range measurement to the first surface interface when the medium of transmission is known and characterized. For instance, transmission of the signal through air to determine the distance from the sensor to a surface. Technology for this purpose has been developed and demonstrated as part of DOE's Robotic Technology Development Program. Extrapolation of the technology to make measurements to the tank floor in a tank containing only liquid is possible. A sensor probe could be deployed within the liquid to sense the range to the floor, and could likely be moved during measurement -- a typical underwater sonar application. Some inaccuracies would be present due to thermal uncertainties and velocity of sound variations due to the liquid composition, but these would probably be minor. However, extracting accurate information for range to a second (and higher) surface interface, as is required to determine waste thickness, is a much more difficult problem.

To determine the waste thickness with ultrasonics would require sonar transmission through the waste material. To do this would probably

\footnotetext{
${ }^{1}$ Memo W. Drotning to $R$. Palmquist $1 / 4 / 94$ re Ultrasonics for Tank Wall Measurement.
} 
require contact between the waste material and the fielded sensor because the impedance mismatch between air and solids would require enormous energy levels to couple any significant sonic power into the transmitted, vs reflected, field. This is the approach taken in Nondestructive Testing measurements, for instance, where the transducer is placed in contact with the solid material to be inspected. Typically this requires a transmission-enhancing medium, like an oil or grease, to provide coupling of the ultrasonic energy from the transducer to the solid under inspection (and back). The impedance mismatch between air and solids is large. One can then use very high frequencies ( $1 \mathrm{MHz}$ or more) to get high resolution distance measurement (thousandths of an inch are achievable). But contact would be required, and this would likely prevent any use in a real-time mode for mapping or motion control. Further, the measurement calculations would be seriously compounded by the lack of knowledge of the velocity of sound in the medium under investigation. A determination of sound velocity would have to be performed using reconstituted retrieved material or a mockup of the expected material's composition. Even so, the actual materials' inhomogeneity, porosity, and property variations throughout the tank would likely be unknown and could seriously affect the measurement accuracy. Also, if the waste coating was not in contact with the tank surface in some areas, a serious inaccuracy would result.

Another approach for the wall/floor range measurement using ultrasonics would be to develop a sensor system which uses a fluid jet to make contact with the waste material, and to couple sonic energy into the fluid jet. This approach has been used for making precise measurements in machining applications using a coolant fluid. The approach would take significant development, and may still be unsuccessful due to the difficulty in transmitting sufficient energy past the first liquid/solid interface to find the coating/wall interface. The problems of velocity calibration, coating porosity, and wall interface uncertainty would still remain.

\section{Capacitance Type Sensors ${ }^{2}$}

Capacitance sensors operate by generating a spatially-resolved electric field and measuring displacement current perturbations resulting from changes in the sensor/robot/waste geometry and the waste composition. The output from the sensor is a complex sinusoidal signal which consists of the in-phase (real) and quadrature (imaginary) impedance components. If a charge amplifier buffering scheme is used, the real component corresponds to the capacitance and the imaginary component is related to the losses or resistive current flow. The sensor impedance is a result of the volume interaction between the sensor electric field and the sludge/hardpan, and will depend on the electrical properties of the waste. The electric field volume is

2 Memo J. Novak to S. Thunborg 1/12/94 re Electric Field Sensors for Residual Waste. 
dependent on the shape and size of the sensor electrodes. In experiments supporting development of the Whole Arm Protection (WHAP)Ref ${ }^{3}$ ) sensor, the electric field volume was considered to be a hemisphere with radius of 10 times the diameter of a circle encompassing the electrode pair. By measuring the real and imaginary components, or impedance, of the resulting sensor signal, information about the shape or composition of the material in the electric field was obtained. However, because both shape and electrical properties affect the sensor measurement, an independent measurement of one is required to permit an accurate assessment of the other.

For all types of material, the sensitivity of the electric field sensor depends on the standoff distance from the material to be measured. For example, if the workpiece is a flat metal plate and the intervening space is filled with air, the losses are very small and the resulting signal predominantly indicates changes in capacitance. This measurement is most sensitive at small distances from the sensor and becomes decreasingly sensitive asymptotically at greater distances. The slope of the capacitance curve is, however, nonzero and measurable up to a distance of about 10 times the diameter of the sensor.

Because the electric field intensity decreases with distance from the electrodes, the device is very sensitive to material close to the electrodes. However, if the sensing electrodes are covered with a thin dielectric material, the electric field properties within the coating can be considered to be constant, and this constant capacitance may be subtracted from the measured sensor reading. In this way, the sensor can be protected from the environment, while still capable of detecting distant objects. The thin coating does not, however, protect from erroneous readings as a result of spray deposits from Waste Dislodging Tools. While a spray coating will further distort the signal the magnitude of the error should not be sufficient to preclude use of capacitance technology during simultaneous operation of a Waste Dislodging Tool. Further developmental testing will be required to evaluate the signal distortion resulting from a thin coating of the waste material.

The electric field does not penetrate the surface of conductive materials. If the sludge/hardpan is highly conductive, the impedance signal will be predominantly capacitive. This capacitance measurement will vary depending on the distance between the sensor and the waste. In this mode, the sensor acts as a proximity detector, and can provide information about the topography of the waste surface, but not about waste thickness. Because metals are highly conductive, this technique might be used to prevent robot collisions with objects such as the tank walls or thermocouple trees in a tank.

3 J. L. Novak and J. T. Feddema, "A Capacitance Based Proximity Sensor for Whole Arm Obstacle Avoidance," Proc. IEEE Int. Conf. on Robotics \& Automation, Nice, France, 1992. 
If the sludge/hardpan is a dielectric and only somewhat conductive, the electric field will penetrate the waste. An electric field sensor can be used to detect a highly conductive metal surface beneath the sludge/hardpan if an independent measurement of the dielectric permittivity and conductivity of the waste can be made. The problem with making independent measurement of dielectric permittivity and conductivity are that the waste properties may vary throughout the tank, and technology to make these measurements at localized areas will need to be developed. To use the electrical data for realtime control of a robot system would require near instantaneous conversion of the electrical data to position data. Technique for fast data conversion will need to be developed. Waste inhomogeneities, such as pockets of liquid, will result in erroneous readings if the volume of the pocket occupies a significant fraction of the effective electric field volume, and if the liquid permittivity is significantly different from the solid waste. For example, if a sensor is $5 \mathrm{~cm}$ diameter, the sensing range can be considered to be a hemisphere approximately $50 \mathrm{~cm}$ in radius and volume of $26000 \mathrm{~cm}^{3}$. In this case, a pocket with a volume of $26 \mathrm{~cm}^{3}(0.1 \%$ volume fraction) would have little effect on the measurement.

In summary, the application of capacitance type sensors for thickness verification depends on the conductivity of the waste. As the water content goes up, the conductivity will increase. Experimentation will be required to determine the effect of water, either in the waste or on the waste, due to the waste dislodging processes.

\section{Inductive Sensors ${ }^{4}$}

Inductive coupling devices are commonly employed in metal detectors used by beachcombers and treasure hunters. The device consists of two coils, usually concentric, and electronics to provide an AC signal to one coil and measure the resulting voltage on the other coil. The voltage output of the secondary coil is a measure of the inductive coupling between the coils. When no metal is present, the two coils are an air core transformer with relatively poor coupling between the coils. When a piece of metal is placed in the proximity of the coils the metal acts as a core for the transformer and the coupling between the two coils increases dramatically, providing a change in output at the secondary coil. Since the coupling between the two coils is through the magnetic fields, ferromagnetic materials produce much larger signals than other metals. The output voltage is a single value, and therefore, the size, shape, and distance to a buried piece of metal cannot be uniquely determined.

The inability to determine range for an inductive sensor when applied in conventional applications would appear to preclude their use in

4 Memo B. Spletzer to S. Thunborg $1 / 19 / 94$ re Use of Inductive Coupling for Residual Waste. 
Underground Storage Tanks. However, UST's have a unique feature in that they have an essentially infinite flat plate of ferrous metal. It is very likely that a closed form solution exists for the amount of coupling between two coils of known geometry and an infinite flat plate. Such a solution could be used to determine coil geometry and sensitivity of the inductive sensor to distance from the plate. Conductivity of the waste between the coils and the flatplate should have little effect on the closed form solution but investigations to determine the potential effect should be conducted. Once designed, the device could be readily calibrated in laboratory conditions to produce a reliable instrument.

If induction data is used for input for realtime control of a robotic arm, procedures and fast data conversion techniques need to be developed to convert inductive data to useful data for robot arm control.

It is likely that the device used for waste thickness verification would differ significantly from commercial metal detectors. A commercial detector is designed to locate small metal objects within a foot or so of the detector. It is specifically designed to cover a reasonable amount of area and provide a strong signal for small metal objects. Coil sizes for commercial detectors range from 6 to 24 inches in diameter with the surface area and effective depth covered increasing with increasing size. Since a detector used for a UST is sensing a very large metal object very close, the coils could be much smaller, perhaps only an inch or so in diameter. The coverage area would be much smaller than that of a commercial device. As a result the sensor could operate much closer to the tank wall without an erroneous reading.

The greatest difficulty with inductive coupling is that the device, to some extent, responds to all nearby metal objects. The presence of a robot manipulator could cause significant errors in the measurement. Normally, nearby metal, such as that used in the device itself, does not present a large problem since it produces a constant amount of coupling resulting in a constant offset voltage on the output coil. In the case of a manipulator, the geometry of the nearby metal is not constant relative to the detector and no simple offset voltage exists. The detector would most likely need to be deployed using a nonmetallic wand (much like a commercial detector) to maintain a suitable distance between the manipulator and the detector. Since the tank bottom is very large and quite close, it seems quite feasible that a separation of a foot or two between the manipulator and the detector would be sufficient. If the interference by the manipulator is too great, calibration of the detector may be required for a number of manipulator positions so that, for any given position, the coupling provided by the manipulator could be estimated and used to adjust the detector reading.

An unknown in the application of Inductive Technology to waste thickness measurement is the effect of rebar in the concrete behind the steel tank liners. The rebar pattern should be repeatable thereby making the effect similar throughout the tank, providing the area of coverage of the inductive sensor is large enough to integrate its effect. Experimentation will be required to determine if rebar is a significant problem. 


\section{Ground Penetrating Radar 5}

Ground Penetrating Radar (GPR) is currently being used in several applications where measuring layer thickness is required. One application is the measurement of asphalt thickness on highways. Another is measuring salt layer thickness at Waste Isolation Pilot Plant (WIPP). GPR systems, specifically called Subsurface Interface Radar (SIR), for applications such as these are available commercially. In the UST application the interface between the waste and tank bottom is very distinctive. Much more distinctive than the interface sought in the above applications.

The problem with GPR is that it does not work when used on conductive mediums. Normally a conductivity of less than 10 millisieman per meter is desirable. Conductivities higher than $10 \mathrm{~ms} / \mathrm{m}$ result in decreasing performance of the GPR. Hardpan waste in the tank is expected to be a salt with a low conductivity, if dry. However, entrained water or water added during retrieval operations may result in a waste with a high conductivity.

To determine the thickness of the waste with GPR will require knowledge of the dielectric constant for the waste material. Consequently, it will be necessary to determine the dielectric constant of the waste material. The dielectric constant should be that of the material representative of material remaining after waste retrieval operations have been conducted. Measurement of a representative dielectric constant could become a problem if the constant varies with location in the tank.

In many GPR applications data interpretation is an extremely difficult problem, usually requiring an expert to interpret results. In systems with a distinctive interface it is much less of a problem. In fact, for the asphalt thickness application, procedures have been developed to provide near real time determination of asphalt thickness.

In summary, GPR can be a viable candidate for use in verifying residual hardpan thickness. However, experimentation will be required to determine the effect of water, either entrained in the waste or in/on the waste due to waste dislodging processes.

\section{Magnetometers $^{6}$}

A magnetometer measures the local magnetic field strength and gradient (or the change in magnetic field). It is able to detect buried ferrous objects by measuring the magnetic field of the object. Multiple measurements of gradient in different directions can give a complete measurement of the magnetic field at the point of measurement. Magnetometers are extremely

5 Telecon Tom Fenner, Geophysical Survey Systems, Inc., S. Thunborg, Sandia $1 / 19 / 94$.

6 Memo B. Spletzer to S. Thunborg $1 / 19 / 94$ re Use of Magnetometers for Residual Waste. 
sensitive to small changes in magnetic fields. They are frequently used to detect buried objects and, in general, are much more sensitive than inductively coupled metal detectors.

Because magnetometers are extremely sensitive, they require precise knowledge of the residual magnet field around an object in order to determine range. The residual magnetism is a function of the level at the time of steel manufacturer and of the induced effect of the environment and around the tank. Residual magnetism may vary from tank to tank and within a single tank. Determination of the magnetic field for each tank to the accuracy required is likely to be such a major problem that it is not practical. Consequently, magnetometers appear to be poorly suited to the measurement of residual waste thickness.

\section{Summary}

All of the technologies discussed require knowledge of certain material properties of the material after waste retrieval operations are initiated. The assumption will then be made that the material properties are constant throughout and between tanks. If the properties are not constant, erroneous data will result. Each technology will need to determine its sensitivity to variations in material properties and the need to determine material property variations throughout a tank.

Of the technologies surveyed, Inductive (Metal Detectors) and GPR appear to be most suitable for use as waste thickness sensors. However, GPR is only suitable if the waste can be shown to have a low conductivity after waste retrieval operations. GPR Technology is probably the closest to being commercially available as shown by its use to determine asphalt thickness. Induction technology will require both experimentation and development to determine electric field coupling of the sensor and with the tank lines.

Ultrasonic systems face a formidable problem in coupling sufficient acoustic energy into the waste so that it will reflect a strong signal from the liner. Capacitance systems face a major development problem in determining dielectric permissivity and conductivity at local areas in the tank. Magnetometers require determination of residual magnetic fields and their variation throughout a tank.

It should be noted that if water jet based Waste Dislodging Tools can be shown to effectively remove all waste without substantial damage to the tank liner, the problem of verification becomes much simpler. In this case an end effector tool with a camera may be sufficient. 


\section{2}

\section{Appendix A}

Given: $\quad$ A 75 foot diatank with 8 feet of solid waste in the bottom of the tank. 6 feet of the 8 feet is soft waste and the bottom 2 feet is hard waste. The tank must be cleaned out so that less than 360 cubic feet of waste remain.

Question: What thickness, $t$, of waste is allowable on the bottom and sides of the tank after cleaning?

Solution: Waste in bottom of tank

$$
V_{1}=\frac{\pi D^{2}}{4} t
$$

Waste on sides of tank

$$
\mathrm{V}_{2}=\pi \mathrm{Dt}(\mathrm{h}-\mathrm{t})
$$

However, since $t$ is very small compared to $h ; V_{2}$ may be rewritten as

$$
\mathrm{V}_{2}=\pi \mathrm{Dth}
$$

Then

$$
\mathrm{V}_{1}+\mathrm{V}_{2}=360 \text { cubic feet }
$$

If $\mathrm{D}=75 \mathrm{ft}$., $\mathrm{h}=8 \mathrm{ft}$.

$t=0.68$ inches

If $\mathrm{D}=75 \mathrm{ft}$., $\mathrm{h}=2 \mathrm{ft}$.

$$
t=0.88 \text { inches }
$$




\section{DISTRIBUTION}

No. of

Copies

2 Oak Ridge National Laboratories Attn: Bill Hamel

2 Oak Ridge National Laboratories Attn: Barry Burks

2 Pacific Northwest Laboratories Attn: Dave Bennett

2 Pacific Northwest Laboratories Attn: Mark Evans

5 Westinghouse Hanford Co. Attn: Pete Gibbons

J. Yount

W. Jaquish

L. McDaniel

A. Williams

10 Westinghouse Hanford Co.

Attn: Eric Shen

1 Lawrence Livermore National Laboratories Attn: Erna Grasz

2 FEMP

Attn: Brack Hazen

Don Herman

2 West Savannah River

Attn: Clyde Ward

Randy Singer

1 WINCO

Attn: Bruce Wilding

1 Los Alamos National Laboratories Attn: R. Hollen

1 DOE - HQ

Lin Yarbrough 


\section{DISTRIBUTION}

No. of

Copies

1 MS 0322 P. J. Eicker, 2100

1 MS 0949 R. W. Harrigan, 2102

1 MS 0951 P. A Erickson, 2103

1 MS 0949 P. A. Molley, 2111

1 MS 0951 D. R. Strip

1 MS 0952 R. D. Palmquist, 2151

1 MS 0952 B. R. Davies, 2151

1 MS 0952 W. E. Ford, 2151

1 MS 0952 R. J. Anderson, 2151

1 MS 0952 D. J. Miller, 2151

60 MS 0952 D. S. Horschel, 2161

1 MS 0952 W. M. Davidson, 2161

1 MS 0952 J. M. Griesmeyer, 2161

1 MS 0952 M. J. McDonald, 2161

1 MS 0952 S. E. Slezak, 2161

1 MS 0952 K. Kwok, 2161

1 MS 1006 P. Garcia, 2171

1 MS 1007 A. T. Jones, 2172

1 MS 9018 Central Technical Files, 8523-2

7 MS 0899 Technical Library, 13414

1 MS 0619 Technical Publications, 13416

10 MS 0100 Document Processing for DOE/OSTI, 7613-2 